\title{
THE
}

7-1-1986

\section{Finite-Size Interaction Amplitudes and their Universality: Exact, Mean-Field, and Renormalization-Group Results}

J. O. Indekeu

M. P. Nightingale

University of Rhode Island, nightingale@uri.edu

W. V. Wang

Follow this and additional works at: https://digitalcommons.uri.edu/phys_facpubs

Terms of Use

All rights reserved under copyright.

\section{Citation/Publisher Attribution}

Indekeu, J. O., Nightingale, M. P., \& Wang, W. V. (1986). Finite-size interaction amplitudes and their universality: Exact, mean-field, and renormalization-group results. Physical Review B, 34(1), 330-342. doi: 10.1103/PhysRevB.34.330

Available at: http://dx.doi.org/10.1103/PhysRevB.34.330

This Article is brought to you for free and open access by the Physics at DigitalCommons@URI. It has been accepted for inclusion in Physics Faculty Publications by an authorized administrator of DigitalCommons@URI. For more information, please contact digitalcommons-group@uri.edu. 


\title{
Finite-size interaction amplitudes and their universality: Exact, mean-field, and renormalization-group results
}

\author{
J. O. Indekeu* \\ Department of Physics, Massachusetts Institute of Technology, Cambridge, Massachusetts 02139 \\ and Instituut voor Theoretische Fysica, Katholieke Universiteit Leuven, 3030 Leuven, Belgium \\ M. P. Nightingale \\ Department of Physics, University of Rhode Island, Kingston, Rhode Island 02881 \\ and Department of Physics, Massachusetts Institute of Technology, Cambridge, Massachusetts 02139 \\ W. V. Wang ${ }^{\dagger}$ \\ Department of Physics, Massachusetts Institute of Technology, Cambridge, Massachusetts 02139
}

(Received 31 January 1986)

\begin{abstract}
We discuss the interaction between interfaces that is mediated by critical fluctuations, and in particular the universality of the corresponding finite-size amplitudes. In the case of the twodimensional Ising model we address the universality with respect to anisotropy. For this purpose we derive the exact free energy of a finite, anisotropic triangular lattice on a cylinder. For the rectangular Ising model we verify universality also with respect to the magnitude of the boundary fields. In mean-field theory we display the mechanism for this universality and for that with respect to the surface coupling enhancement. Numerical results, which are of experimental relevance, are obtained employing a renormalization-group approximation for three-dimensional systems.
\end{abstract}

\section{INTRODUCTION AND SUMMARY}

In various physical systems ${ }^{1-6}$ of experimental and theoretical interest, correlated fluctuations mediate interface-interface interactions that decay as an inverse power of distance. In the example we consider in detail in this paper, the fluctuating medium is a fluid or solid at an Ising critical point. In this context one may think of the attraction between identical parallel plates immersed in a critical fluid, or of the interaction governing the thickness of a partial wetting layer of a phase that is critical in bulk. Some of our results are expected to be valid also in the case of the $X Y$ transition, of which the $\lambda$ transition in superfluid helium is the paradigm, and in the case of transitions in other universality classes.

One of the most intriguing aspects of these finite-size interactions is the mounting evidence $e^{2,3,5-8}$ of the universality of their amplitudes measured in units of $k_{B} T$. To be more precise, in the Ising universality class in three dimensions, e.g., there are at most eleven different amplitudes. They are associated with systems with different types of surfaces, as characterized by the presence or absence of symmetry breaking fields, and the degree of enhancement of the interactions at the surface.

In order to discuss the scaling theory of the interaction between surfaces, we consider a lattice in $d$ dimensions consisting of $z$ layers of $n \times n \times \cdots(d-1$ times $)$ sites each. Layers 1 and $z$ are top and bottom surfaces of the slab; each of the layers is assumed to be macroscopic in size $(n>>)$. The total free energy measured in units of $k_{B} T$ is written as

$$
F=-\ln Z \text {, }
$$

where $Z$ is the partition function. The surface free energy per column is defined as

$$
f_{S}=\lim _{n \rightarrow \infty}\left(F-z n^{d-1} f_{B}\right) / n^{d-1},
$$

where $f_{B}$ is the bulk free energy per lattice site (per unit volume in the continuum case). We remark that with this definition the surface free energy is singular at the critical point of the $d$-dimensional bulk, where the layer itself, strictly speaking, is noncritical: In general, a thick layer will be critical close to, but not at, bulk criticality. However, our definition is the appropriate one for describing equilibrium between the slab and a bulk reservoir.

We can write

$$
f_{S}=f_{1}+f_{2}+\delta f\left(z, h_{1}, h_{2}, v_{1}, v_{2}\right) \text {, }
$$

where $f_{i}(i=1,2)$ is the surface free energy per surface site in the limit of a semi-infinite system $(z \rightarrow \infty)$. We have allowed for the presence of surface ordering fields $h_{i}$ and surface coupling enhancements $v_{i}$. The term $\delta f$, which vanishes for $z \rightarrow \infty$, represents the finite-size interaction free energy between the two surfaces. If the bulk is criti$\mathrm{cal}$, then the following finite-size homogeneity relation holds for the singular part ${ }^{9}$ of the interaction free energy:

$$
\delta f_{\text {sing }}\left(z, h_{i}, \delta v_{i}\right)=z^{1-d} X\left(z^{y_{H}^{\prime}} h_{i}, z^{y_{i}^{\prime}} \delta v_{i}\right) .
$$

The $\delta v_{i}$ denote the scaling fields $v_{i}-v_{i}^{*}$ that describe the deviations of the surface enhancements away from their fixed-point values. As usual, we distinguish (in dimensions $d>2$ ) the fixed points associated with the ordinary $(O)$, the surface-bulk multicritical, or, in other words, special (SB), and extraordinary $(E)$ surface transitions. ${ }^{10}$ The 
critical exponent $y_{H}^{\prime}$ is the scaling index of the surface ordering field. The exponents $y_{i}^{\prime}$ are the scaling indices of the surface enhancements. They are relevant only at the SB transition. Here we shall consider only the thick-film limits $(z \rightarrow \infty$, all other parameters fixed) of the crossover function $X$. The values of $X$ at the various fixed points, i.e., the finite-size interaction amplitudes, are denoted $\Delta_{\beta_{1}, \beta_{2}}$, where $\beta_{i}=+$ if $h_{i}>0, \beta_{i}=-$ if $h_{i}<0, \beta_{i}=O$ at the ordinary transition, $\beta_{i}=\mathrm{SB}$ at the surface-bulk multicritical point, and $\beta_{i}=E$ at the extraordinary transition. Obviously, in the latter three cases, $h_{i}=0$.

The remainder of this paper is organized as follows. In Sec. II we present results of exact calculations for the two-dimensional, triangular Ising model, and for the rectangular model with surface fields. In Sec. III we discuss the finite-size amplitudes in mean-field theory. Finally, Sec. IV is devoted to a Migdal-Kadanoff renormalization-group calculation of these quantities. Now we proceed to present an overview of the main results, some of which were reported in a previous publication. ${ }^{5}$ Very recently, finite-size amplitudes in $d=2$ have been related to the central charge of the conformal algebra. ${ }^{11}$ These results agree with the exact results reported in Ref. 5 .

For the anisotropic triangular Ising model we have calculated exactly the surface free energy of a finite cylinder. The dominant finite-size correction for a cylinder with infinite perimeter and finite height $z$ decays with $1 / z$, as predicted by finite-size scaling. We obtain the amplitude $\Delta_{O, O}=-\pi / 48$, and verify that it is universal with respect to the lattice anisotropy, provided that the cylinder is rescaled so as to render the decay of pair correlations isotropic at large distances. Note that this provides an explicit verification and generalization of assumptions that underlie results obtained in Ref. 11 . Our result is more general in the sense that in addition to a rescaling of one of the two principal axes (space-space or space-time), also a rotation may be needed to restore universality. In the special case of the isotropic square lattice, this agrees with a result of Au-Yang and Fisher. From their expression for the rectangular strip with boundary fields $\left(h_{1} h_{2} \geq 0\right)$ we derive $\Delta_{O,+}=\pi / 24, \Delta_{+,+}=-\pi / 48$, and explicitly check the irrelevance of the magnitude of the surface fields, and of the lattice anisotropy in the same sense as above. We further conjecture that $\Delta_{+,-}=2^{d} \Delta_{O,+}=\pi / 6$ (also see mean-field results below). ${ }^{12}$

Within mean-field theory we obtain the orderparameter profiles that minimize the free-energy functional per unit area of a slab. It turns out that only two profiles exist in the scaling limit. Truncated appropriately they satisfy the various boundary conditions at the slab surfaces. Our study suggests that, in general, like boundaries attract $(\Delta<0)$, but unlike boundaries repel $(\Delta>0){ }^{13}$ Universality with respect to the magnitude of surface fields and surface-coupling enhancements holds, but the amplitudes do depend on the spin quantum number (which we have taken to be $\frac{1}{2}$ ), and on the lattice coordination number $q$, in this approximation. We obtain that $\Delta_{O, O}, \Delta_{O, \mathrm{SB}}$ and $\Delta_{\mathrm{SB}, \mathrm{SB}}$ all vanish, which reflects that fluctuations have been ignored. The other amplitudes are nonzero, and we find $\Delta_{O, E} \cong 11.82 / q^{2}$. Furthermore, we find that the following equalities hold:

$$
\Delta_{\beta, E}=\Delta_{\beta,+} \text { if } \beta=O, \mathrm{SB}, E \text {, or }+ \text {. }
$$

These equalities are physically quite obvious, and most likely of general validity even beyond mean-field theory. Also, we obtain the equality ${ }^{12}$

$$
\Delta_{O,+}=2^{-d} \Delta_{+,-},
$$

with $d=4$. Furthermore,

$$
\Delta_{\mathrm{SB},+}=2^{-d} \Delta_{+,+},
$$

with $d=4$. Finally,

$$
\Delta_{+,+}=-\frac{d}{16} \Delta_{+,-} .
$$

In mean-field theory this holds with $d=4$, but also the exact results in two dimensions satisfy this relation.

To see how fluctuations affect the amplitudes of the finite-size interaction and to obtain estimates in three

TABLE I. Migdal-Kadanoff renormalization-group estimates for the finite-size interaction amplitudes $\Delta$ corresponding to the ordinary $(O)$, surface-bulk $(\mathrm{SB})$ for $d>2$, and extraordinary $(E)$ transitions of a surface without boundary fields, and as a function of the signs of boundary fields. Space dimensionality $d$ equals 2,3 , or 4 . Numbers in parentheses are exact, conjectured, or from $\epsilon$ expansion. Furthermore, $\Delta_{O, E}=\Delta_{O,+}, \Delta_{\mathrm{SB}, E}=\Delta_{\mathrm{SB},+}, \Delta_{E, E}=\Delta_{E,+}=\Delta_{+,+}$.

\begin{tabular}{cccccccc}
\hline \hline$d$ & $\Delta_{O, O}$ & $\Delta_{O, \mathrm{SB}}$ & $\Delta_{\mathrm{SB}, \mathrm{SB}}$ & $\Delta_{O,+}$ & $\Delta_{\mathrm{SB},+}$ & $\Delta_{+,+}$ & $\Delta_{+,-}$ \\
\hline 2 & -0.055 & & & 0.130 & & 0 & 0.881 \\
& $(-0.065)^{\mathrm{a}}$ & & & $(0.131)^{\mathrm{b}}$ & & $(-0.065)^{\mathrm{b}}$ & $(0.524)^{\mathrm{c}}$ \\
3 & $\begin{array}{c}-0.015 \\
(-0.023)^{\mathrm{d}}\end{array}$ & 0.017 & 0.019 & 0.051 & 0.017 & 0 & 0.279 \\
4 & -0.007 & -0.003 & $\cong 10^{-5}$ & 0.017 & 0.012 & 0 & 0.100 \\
\hline
\end{tabular}

${ }^{\mathrm{a}}$ Reference 2, exact.

${ }^{b}$ Reference 5, exact.

${ }^{c}$ References 2 and 5 , conjectured.

${ }^{\mathrm{d}}$ Reference 3, $\epsilon$ expansion. Systematic expansion of first order in $\epsilon=4-d$ yields -0.023 , whereas use of Eqs. (4.4) and (4.9) in Ref. 3 at $\epsilon=1$ yields -0.101 .

${ }^{\text {e}}$ Reference 3, exact. 
dimensions, we have performed a Migdal-Kadanoff renormalization-group calculation. Universality with respect to all surface parameters arises quite naturally. In Table I we present the numerical results. Equalities (1.5) hold whenever applicable $(d>2)$. The numerical performance of the Migdal-Kadanoff approach is surprisingly good, as comparison shows with exact values in $d=2$ and 4 (the numbers in parentheses in Table I). A further test of the accuracy in $d=3$ is provided by a comparison with a recent Monte Carlo computation, where a finite-size amplitude related to, but different from $\Delta$, was computed (see Sec. IV for details). Again, the agreement is excellent.

A final remark concerns the comparison of the meanfield-theory amplitudes with those obtained with the renormalization approach in $d=4$. Taking the coordination number $q=2 d$ (hypercubic lattice), we conclude that the mean-field results are typically an order of magnitude larger.

\section{ANISOTROPIC, TRIANGULAR ISING MODEL}

In this section we investigate the universality of the amplitude of the finite-size interaction between the surfaces of the exactly solvable, two-dimensional, anisotropic Ising model on a triangular lattice. Of course, this includes the square and rectangular lattices as special cases. First, we discuss the effect of anisotropy on this universality. Then we present the results of the analytic calculations for this model. Finally, in the case of the rectangular lattice we also present results for the universality with respect to the magnitude of the boundary fields.

We consider a lattice system bounded by two infinite surfaces a finite distance apart. What is the effect of anisotropy in the coupling constants on the finite-size interaction between the two surfaces? If the interactions perpendicular to the free surface are made weaker, while at the same time those parallel to the surface are strengthened to maintain criticality, the effective distance between the surfaces increases in the sense that correlations between them are expected to decrease. This leads to a smaller finite-size amplitude. In other words, the finite-size amplitude is unlikely to be universal, if the distance between the surfaces is expressed in units of the lattice spacing. However, one would expect universality to be restored-and this is corroborated by our analytical calculations-if the units of length, used in the definitions of the thickness of the system and of the surface free energy per unit length (in two dimensions), are chosen in a way such that isotropy is restored to the bulk correlations at large distances in these units. In two dimensions, where contours of constant correlation are expected to be ellipses, this implies, as discussed in detail below, that the finite-size amplitude is a universal number times a function that depends on the eccentricity and on the orientation of the principal axes.

We consider a finite lattice ( $n$ rows and $m$ columns) of Ising spins $s_{j, k}=1, j=1, \ldots, n$ and $k=1, \ldots, m$, with cylindrical boundary conditions: periodic in the $k$ direction, $s_{j, 1}=s_{j, m+1}$ and with free surfaces at $j=1$ and $j=n$. The Hamiltonian, divided by $-k_{B} T$, reads

$$
\begin{aligned}
H= & K_{1} \sum_{j=1}^{n} \sum_{k=1}^{m} s_{j, k} s_{j, k+1}+K_{2} \sum_{j=1}^{n-1} \sum_{k=1}^{m} s_{j, k} s_{j+1, k} \\
& +K_{3} \sum_{j=1}^{n-1} \sum_{k=1}^{m} s_{j, k} s_{j+1, k+1} .
\end{aligned}
$$

For simplicity we assume all interactions to be ferromagnetic $\left(K_{i}>0\right)$.

The total free energy (in units of $k_{B} T$ ) $F=n m f=-\ln Z$ is expected to be of the form

$$
F=n m f=n m f_{\infty}+m\left(f_{1}+f_{2}\right)+m \delta f,
$$

where $f_{\infty}$ is the free energy per spin and $f_{1}+f_{2}$ is the excess free energy per column in the thermodynamic limit, while $\delta f$ contains the finite-size contributions, again in units of $k_{B} T$. For $m \rightarrow \infty, \delta f$ is of the form

$$
\delta f=\Delta_{o, o} n^{-1}+o\left(n^{-1}\right) .
$$

Using standard techniques, ${ }^{14}$ the free energy of the triangular lattice can be calculated, and the finite-size amplitude can be extracted (see Appendix A 1). We find

$$
\Delta_{O, O}=-\frac{\pi}{48} \eta,
$$

where

$$
\eta=\frac{C_{2}^{2}}{S_{1}+S_{2}}
$$

with $C_{i}=\cosh \left(2 K_{i}\right)$ and $S_{i}=\sinh \left(2 K_{i}\right)$, where the $S_{i}$ and $C_{i}$ are related via the condition of criticality $S_{1} S_{2}$ $+S_{2} S_{3}+S_{3} S_{1}=1 .{ }^{15}$ In the special case of the square lattice $\left(K_{1}=K_{2}\right.$ and $\left.K_{3}=0\right)$ the right-hand side of expression (2.4) reduces to $-\pi / 48$. We now show that the factor $C_{2}^{2} /\left(S_{1}+S_{2}\right)$ indeed has the geometric interpretation discussed above.

In order to do this we introduce an anisotropic metric that renders correlations isotropic as follows. Denote by $\xi_{i}$ the correlation length measured in units of the lattice constant in the direction of the bonds with interaction strength $K_{i}$. Upon approaching the critical point, these correlation lengths diverge, but ratios $\kappa_{i}=\xi_{3} / \xi_{i}$ remain finite and precisely define the anisotropy that is relevant here. One finds (see Appendix A 2)

$$
\begin{aligned}
& \kappa_{3}=1, \\
& \kappa_{2}=C_{1} /\left(S_{1}+S_{2}\right), \\
& \kappa_{1}=C_{2} /\left(S_{1}+S_{2}\right) .
\end{aligned}
$$

We define the $\delta$ distance from a point $(x, y)$ in the lattice to the origin as $\delta^{2}(x, y)=\alpha x^{2}+2 \beta x y+\gamma y^{2}$. Here, $\alpha, \beta$, and $\gamma$ are determined so that the contours of equal correlation are circles in this metric. This requires that

$$
\delta^{2}\left(1 / \kappa_{1}, 0\right)=\delta^{2}\left(0,1 / \kappa_{2}\right)=\delta^{2}\left(-\kappa_{3}^{-1}, \kappa_{3}^{-1}\right)=1,
$$

so that $\alpha=\kappa_{1}^{2}, \gamma=\kappa_{2}^{2}$, and $\beta=\frac{1}{2}\left(\kappa_{1}^{2}+\kappa_{2}^{2}-1\right)$.

Converting from the surface free energy per column to a free energy per unit $\delta$ length, and also measuring the width of the strip in these $\delta$ units, the finite-size amplitude changes to $R \Delta_{O, O}$, where $R=\left[\gamma / \alpha-(\beta / \alpha)^{2}\right]^{1 / 2}$ is the $\delta$ distance between rows divided by the $\delta$ distance be- 
tween neighboring lattice sites at the boundary. Since it follows from Eqs. (2.6) that $R=\left(S_{1}+S_{2}\right) / C_{2}^{2}$, the finitesize interaction using the appropriate units indeed is universal for the anisotropic triangular Ising model.

Two special cases deserve mentioning. Firstly, for the isotropic triangular lattice one has $S_{i}^{2}=\frac{1}{3}$ at criticality, so that $\eta=2 \sqrt{3} / 3$, which is the expected ratio of nearestneighbor and row-row distances of the undistorted triangular lattice. Secondly, for the rectangular lattice $S_{3}=0$, and $S_{1} S_{2}=1$. In this case $\eta=1 / R=\left(S_{2} / S_{1}\right)^{1 / 2}$. This turns out to be the ratio of the correlation lengths perpendicular and parallel to the strip, which is precisely the geometric ratio needed to restore isotropy of correlations in this case.

Finally, for the rectangular lattice we derived the finite-size amplitudes from the expression for the free energy of an infinite strip with boundary fields, as given by Au-Yang and Fisher (second of Ref. 2). We find here explicitly that in the thick-film limit only the signs of the fields are relevant. The results are $\Delta_{+,+}=\Delta_{O, O}$ $=-\eta \pi / 48$ and $\Delta_{o,+}=\eta \pi / 24$. This implies that universality is regained also in the presence of boundary fields upon rescaling lengths in the physically obvious way.

\section{MEAN-FIELD THEORY}

In this section we obtain estimates of the amplitudes of the finite-size contribution to the surface free energy of a system of finite thickness employing a Landau theory at bulk criticality. The free energy per unit area of an infinite slab is obtained as the minimum of a functional of the form

$$
F=\int_{l_{1}}^{l_{2}} \mathscr{F}(M, \dot{M}) d x+\sum_{i=1}^{2} F_{i}\left(M\left(l_{i}\right)\right),
$$

where $M=M(x)$ is a magnetization profile which varies across the slab $l_{1} \leq x \leq l_{2} ; \dot{M}=d M / d x$. The functions $\mathscr{F}$ and $F_{i}$ will be specified below.

To find the free energy per unit area as a function of the thickness $z=l_{2}-l_{1}$ we consider the change of the free energy, $\delta F$, upon varying $M \rightarrow M+\delta M, l_{i} \rightarrow l_{i}+\delta l_{i}$ :

$$
\begin{aligned}
\delta F= & \int_{l_{1}}^{l_{2}}\left(\frac{\partial \mathscr{F}}{\partial M}-\frac{d}{d x} \frac{\partial \mathscr{F}}{\partial \dot{M}}\right) \delta M d x \\
& +\left(\frac{\partial \mathscr{F}}{\partial M}(\Delta M-\dot{M} \delta l)+\mathscr{F} \delta l\right]_{l_{1}}^{l_{2}}+\sum_{i=1}^{2} \frac{\partial F_{i}}{\partial M} \Delta M_{i}
\end{aligned}
$$

where $\Delta M=\dot{M} \delta l+\delta M$.

The equilibrium profile satisfies the equations

$$
\begin{aligned}
& \frac{\partial \mathscr{F}}{\partial M}-\frac{d}{d x} \frac{\partial \mathscr{F}}{\partial \dot{M}}=0, \quad l_{1} \leq x \leq l_{2} \\
& -\frac{\partial \mathscr{F}}{\partial \dot{M}}+\frac{\partial F_{1}}{\partial M}=0, \quad x=l_{1} \\
& \frac{\partial \mathscr{F}}{\partial \dot{M}}+\frac{\partial F_{2}}{\partial M}=0, \quad x=l_{2}
\end{aligned}
$$

as follows from variations with $\delta l_{1}=\delta l_{2}=0$.
Equation (3.3) has a first integral,

$$
\frac{\partial \mathscr{F}}{\partial \dot{M}} \dot{M}-\mathscr{F}=E
$$

with $E$ a constant for given thickness $z$.

It also follows that $\delta_{0} F$, the change in the equilibrium free energy per unit area, i.e., for a profile satisfying Eqs. (3.3) and (3.4), due to variations $\delta l_{i}$, is given by

$$
\delta_{0} F=-E \delta z
$$

We consider the case

$$
\begin{aligned}
& \mathscr{F}=\frac{1}{2} C \dot{M}^{2}+\frac{1}{4} B M^{4}, \\
& F_{i}=\frac{1}{2} V_{i} M^{2}-H_{i} M .
\end{aligned}
$$

The absence of a term quadratic in $M$ in (3.7) reflects the fact that the system is at bulk criticality. The boundary fields are $H_{i}$ in (3.8) and we allow different surfacecoupling enhancements $V_{i}$ for both surfaces. With (3.7) and (3.8), (3.5) and (3.4) assume the form

$$
\begin{aligned}
& \dot{m}^{2}-m^{4}=E /|E|, \\
& \dot{m}-v_{1} m+h_{1}=0, \quad x=l_{1}, \\
& \dot{m}+v_{2} m-h_{2}=0, \quad x=l_{2},
\end{aligned}
$$

where $\quad m=(B / 4|E|)^{1 / 4} M, \quad \zeta=\left(B|E| / C^{2}\right)^{1 / 4} x$, $\dot{m}=d m / d \zeta=[C /(2|E|)]^{1 / 2} \dot{M}, \quad v_{i}=\left(B C^{2}|E|\right)^{-1 / 4} V_{i}$, and $h_{i}=(2 C|E|)^{-1 / 2} H_{i}$.

We shall employ the following two solutions of (3.9). For $E>0$ the inverse profile $\xi=\xi(m)$ is given by

$$
\zeta=\int_{0}^{m}\left(1+v^{4}\right)^{-1 / 2} d v
$$

corresponding to an antisymmetric profile [Fig. 1(a)]. The reduced magnetization $m$ diverges at

$$
\pm \zeta=K_{a} \equiv \int_{0}^{\infty}\left(1+v^{4}\right)^{-1 / 2} d v \text {. }
$$

Close to these boundaries the right-hand side of (3.9) is negligible so that

$$
m \cong \pm\left(K_{a} \mp \zeta\right)^{-1}, \quad 0<K_{a} \mp \zeta \ll 1 .
$$

Close to $\zeta=0$, on the other hand, we can neglect $m^{4}$ in (3.9), so that

$$
m \cong \xi, \quad|\zeta| \ll 1
$$

The approximate forms (3.13) and (3.14) will be used in the analysis of the various boundary conditions.

For $E<0$, one has a solution

$$
|\zeta|=\int_{1}^{m}\left(v^{4}-1\right)^{-1 / 2} d v
$$

corresponding to a symmetric profile [Fig. 1(b)] which close to

$$
\pm \zeta=K_{s} \equiv \int_{1}^{\infty}\left(v^{4}-1\right)^{-1 / 2} d v
$$

can be approximated by

$$
m \cong\left(K_{s} \mp \zeta\right)^{-1}, \quad 0<K_{s} \mp \zeta \ll 1 .
$$

For small $|\zeta|$, on the other hand, one has 


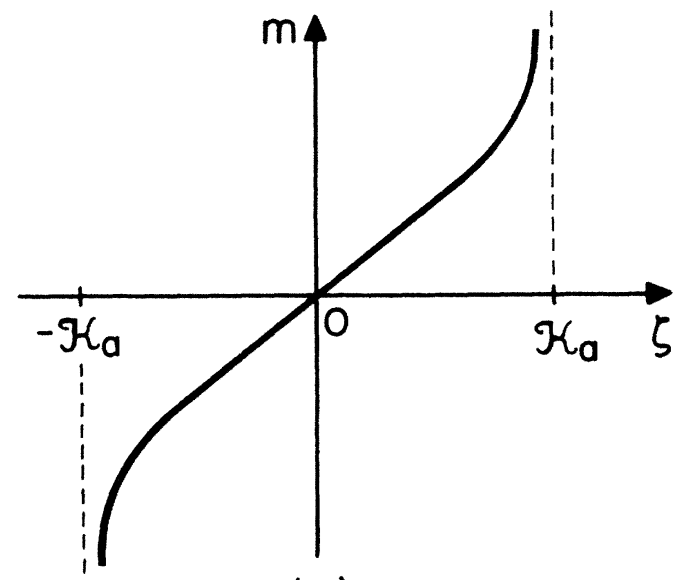

(a)

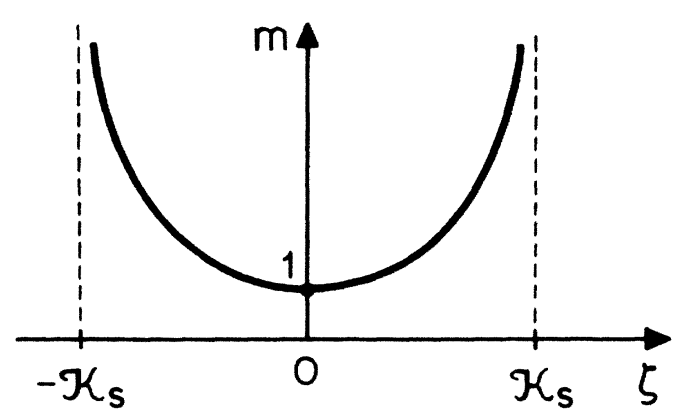

(b)

FIG. 1. Mean-field-theory magnetization profile in reduced units: (a) $E>0$, Eq. (3.11); (b) $E<0$, Eq. (3.15).

$$
m \cong 1+\zeta^{2}, \quad|\zeta| \ll 1 .
$$

We shall consider four separate situations: (1) $h_{1} h_{2}<0$, (2) $h_{1} h_{2}>0$, (3) $h_{1} \neq h_{2}=0$, and (4) $h_{1}=h_{2}=0$.

\section{A. Case 1: $h_{1} h_{2}<0$}

Without loss of generality we choose $h_{1}<0$. A profile satisfying the boundary conditions can be found in this case by truncation of the antisymmetric profile given by (3.11), at $\zeta=-K_{a}+\delta l_{1}$ and $\zeta=K_{a}-\delta l_{2}$, respectively. Substituting the expressions (3.13) into the boundary conditions (3.10), one finds

$$
\delta l_{i}=\left[v_{i}+\left(v_{i}^{2}+4\left|h_{i}\right|\right)^{1 / 2}\right] / 2\left|h_{i}\right|
$$

which can be done consistently as long as $\delta l_{i} \ll<1$, i.e.,

$$
\left|h_{i}\right| \gg 1+v_{i} \text {. }
$$

Under these conditions the width of the system in reduced units differs negligibly from $2 K_{a}$.

Expressing this result in the original units and employing (3.6), we find that the finite-size interaction decays as $z^{-3}$ to leading order, as finite-size scaling would predict in $d=4$. The interaction amplitude is independent of the boundary fields and is given by

$$
\Delta_{+,-}=16 K_{a}^{4} C^{2} / 3 B \text {. }
$$

\section{B. Case 2: $h_{1} h_{2}>0$}

We choose $h_{1}>0$. The only difference with case 1 is that the boundary conditions can now be satisfied only by truncating the symmetric profile (3.15). The result is

$$
\Delta_{+,+}=-\frac{16}{3} K_{s}^{4} C^{2} / B \text {, }
$$

under the same conditions (3.20).

$$
\text { C. Case 3: } h_{1} \neq h_{2}=0
$$

The following subcases have to be treated separately: (a) ordinary surface transition $\left(v_{2}>0\right)$; (b) surface-bulk multicritical point $\left(v_{2}=0\right)$; (c) extraordinary surface transition $\left(v_{2}<0\right)$.

(a) Ordinary $\left(v_{2}>0\right)$. Choosing $h_{1}<0$, the antisymmetric profile can be truncated at $\zeta=-K_{a}+\delta l_{1}$ and $\zeta=-\delta l_{2}$. As above, expression (3.19) holds, but now only for $\delta l_{1}$. For $\delta l_{2}$ we find, substituting (3.14) into (3.10),

$$
\delta l_{2}=v_{2}^{-1} \text {. }
$$

From $\left|\delta l_{2}\right| \ll 1$ it follows that

$$
\Delta_{O,+}=K_{a}^{4} C^{2} / 3 B
$$

independent of $h_{1}$ and $v_{1}$.

(b) Surface-bulk $\left(v_{2}=0\right)$. Choosing $h_{1}>0$, the symmetric profile truncated at $\xi=-K_{s}+\delta l_{1}$ and $\xi=0$, respectively, satisfies the boundary conditions so that

$$
\Delta_{\mathrm{SB},+}=-K_{s}^{4} C^{2} / 3 B
$$

(c) Extraordinary $\left(v_{2}<0\right)$. The profile found in (a) satisfies both (3.9) and (3.10), but has to be rejected as a solution that maximizes the free energy, since it has the unphysical feature that a boundary field of one sign on one side of the system induces a magnetization of opposite sign on the other. Apparently, the symmetric profile must be truncated at $\zeta=-K_{s}+\delta l_{1}$, with $\delta l_{1}$ given by (3.19), and $\zeta=K_{s}-\delta l_{2}$, with

$$
\delta l_{2}=-\left|v_{2}\right|^{-1}
$$

so that

$$
\Delta_{E,+}=-16 K_{S}^{4} C^{2} / 3 B
$$

$$
\text { D. Case 4: } h_{1}=h_{2}=0
$$

In this case, it is relevant whether the values of $v_{i}$ on both surfaces are the same or different. We find that the magnetization vanishes, and therefore no interaction results, whenever $v_{1} \geq 0$ and $v_{2} \geq 0$. This lack of interaction is a consequence of neglecting the fluctuations in the mean-field approximation. On the other hand, when $v_{1}<0$ and $v_{2}<0$, we find that the symmetric profile must be truncated at $\zeta=-K_{s}+\delta l_{1}$, with $\delta l_{1}=\left|v_{1}\right|^{-1}$, and at $\xi=K_{s}-\delta l_{2}$, with $\delta l_{2}=\left|v_{2}\right|^{-1}$. This reflects that the 
spontaneous magnetizations have the same signs on both surfaces, so that

$$
\Delta_{O, E}=\Delta_{+,+} \text {. }
$$

Next, considering $v_{1}<0$ and $v_{2}=0$, we must again invoke the symmetric profile, but now truncated at $\zeta=-K_{s}+\delta l_{1}$, with $\delta l_{1}=\left|v_{1}\right|^{-1}$, and at $\zeta=0$, so that

$$
\Delta_{\mathrm{SB}, E}=2^{-4} \Delta_{+,+} \cdot
$$

Finally, examining $v_{1}<0$ and $v_{2}>0$, the antisymmetric profile applies, truncated at $\zeta=-K_{s}+\delta l_{1}$, with $\delta l_{1}$ $=\left|v_{1}\right|^{-1}$, and at $\zeta=-\delta l_{2}$, with $\delta l_{2}=v_{2}^{-1}$, so that

$$
\Delta_{O, E}=2^{-4} \Delta_{+,-} \text {. }
$$

Before presenting the numerical results, we remark the following concerning the connection between the Landau theory developed above and the mean-field theory (as distinguished in Ref. 16).

In the mean-field theory, (3.7) is modified to contain higher-order terms, and takes the form (at $T=T_{c}$ )

$$
\mathscr{F}=\frac{1}{2} C \dot{M}^{2}+\frac{1}{4} B M^{4}+O\left(M^{6}\right),
$$

where the higher-order terms in $M$ result in a bounded magnetization. We show that the finite-size amplitudes are insensitive to these higher-order terms. The inverse profiles (3.11) and (3.15) are not significantly modified for sufficiently large $z$. On the basis of (3.31), (3.9) becomes

$$
\dot{m}^{2}-m^{4}-O\left(M^{6}\right) /|E|=E /|E|,
$$

so that the antisymmetric inverse profile changes to

$$
\widetilde{\zeta}=\int_{0}^{m}\left[1+v^{4}+O\left(|E|^{3 / 2} v^{6}\right) /|E|\right]^{-1 / 2} d v,
$$

which yields

$$
\widetilde{\zeta}=\zeta(m)+O\left(z^{-1}\right),
$$

where $\zeta(m)$ is the inverse Landau profile defined in (3.11), and we have used $m \sim|E|^{-1 / 4}$ and $|E| \sim z^{-4}$.

For the symmetric profile the same result (3.33) holds, as one verifies easily.

We can convert the expressions for the $\Delta$ 's into numbers, taking $B=\frac{1}{3}$ for Ising spin- $\frac{1}{2}$ particles, and $C^{2}=q^{-2}$, where $q$ is the lattice coordination number. Here we have followed Nakanishi and Fisher. ${ }^{16,17}$ We obtain, for example

$$
\Delta_{O, E} \cong 11.82 / q^{2}
$$

The other amplitudes are either zero or simply related to this one via the equalities mentioned above, and also the following equality,

$$
\Delta_{+,+}=-\Delta_{+,-} / 4,
$$

which relies on the fact that $K_{a}^{2}=2 K_{s}^{2}$.

In terms of complete elliptic integrals of the first kind, $F(\varphi, a)$, we have

$$
K_{a}=\frac{1}{2} F(\pi, \pi / 4)=F(\pi / 2, \pi / 4) \cong 1.854
$$

and

$$
K_{s}=(\sqrt{2} / 2) F(\pi / 2, \pi / 4) \cong 1.311 .
$$

The present results extend and also correct the mean-field theory amplitudes given in Ref. 5 .

Unsatisfactory features of the mean-field results are the absence of finite-size interaction for many cases with zero fields $\left(h_{1}=h_{2}=0\right)$, and the dependence of the amplitudes on spin (through $B$ ) and lattice coordination number (through $C^{2}$ ).

A last remark we want to make is that, in the Landau theory studied by Lipowsky and Seifert, ${ }^{4}$ the finite-size interaction between a wall and the interface between a nearcritical wetting layer and a bulk vapor phase (spectator phase) can be calculated in complete analogy with the calculations described above. ${ }^{18}$ It is seen that our interaction energy $E$ corresponds to their scaling field $t$ describing the deviation from critical-endpoint coexistence. At coexistence the wetting layer would have infinite thickness and represent a critical bulk phase. Away from coexistence, we obtain ${ }^{18}$ the layer thickness $z$ explicitly as a function of $t$. Furthermore, as far as the calculation of the finite-size amplitude is concerned, the spectator phase can simply be replaced by the boundary field $h_{2}$ acting at the interface between the wetting layer and the vapor phase. From the definition of the bulk potential, ${ }^{4}$ it is clear that $h_{2}=\xi^{-2}$, where $\xi$ is the bulk correlation length in the vapor phase.

\section{MIGDAL-KADANOFF RENORMALIZATION-GROUP APPROXIMATION}

Consider a $d$-dimensional Ising model on a hypercubic lattice consisting of $z$ layers of $n \times n \times n \cdots(d-1$ times) sites each. The interactions are between nearest neighbors: surface coupling $K_{S}=J_{S} / k_{B} T$ within the two surface layers, and bulk coupling $K_{B}=J_{B} / k_{B} T$. Surface fields $h_{1}$ and $h_{2}$ act on top- and bottom-layer spins, respectively.

To obtain the surface free energy per column, $f_{S}$, as defined in (1.2), we first compute the bulk free energy per spin, $f_{B}$. Performing a Migdal-Kadanoff (bond-moving) renormalization-group (RG) transformation ${ }^{19,20}$ with length rescaling $b$, one finds

$$
\begin{aligned}
-f_{B}-d K_{B}= & \ln 2\left[b^{d}-1-d(b-1)\right] /\left(b^{d}-1\right) \\
& +d b^{-d} \sum_{k=0}^{\infty} b^{-d k_{B}^{(k)}} .
\end{aligned}
$$

The term $-d K_{B}$ is the reduced energy per spin in a configuration of complete spin alignment. It occurs separately because we choose to work with a normalized ${ }^{21}$ Boltzmann weight $\exp \left[K_{B}\left(s_{i} s_{j}-1\right)\right]$ for each pair of nearest-neighbor spins $s_{i}, s_{j}$. The term proportional to $\ln 2$ results from summing over the dangling spins that remain after bond moving. ${ }^{22}$ The infinite sum features regular functions $g_{B}^{(k)}$, where $k$ refers to the $k$ th $\mathrm{RG}$ iteration. Explicitly,

$$
g_{B}^{(k)}=\ln \left\{\frac{1}{2}\left[\left(1+\tilde{\rho}_{B}^{(k)}\right)^{b}+\left(1-\tilde{\rho}_{B}^{(k)}\right)^{b}\right]\right\},
$$

where

$$
\begin{aligned}
& \rho_{B}^{(k)} \equiv\left[\exp \left(-2 K_{B}\right)\right]^{(k)}, \\
& \tilde{\rho}_{B}^{(k)} \equiv\left(\rho_{B}^{(k)}\right)^{b^{d-1}},
\end{aligned}
$$


and

$$
\rho_{B}^{(k+1)}=\frac{\left(1+\tilde{\rho}_{B}^{(k)}\right)^{b}-\left(1-\tilde{\rho}_{B}^{(k)}\right)^{b}}{\left(1+\tilde{\rho}_{B}^{(k)}\right)^{b}+\left(1-\widetilde{\rho}_{B}^{(k)}\right)^{b}} .
$$

We proceed to compute $F$, the total free energy of the slab of $z$ layers. For fixed rescaling $b$, we restrict the values of $z$ to $b^{m}+1$, where $m$ is integral. This restric- tion becomes unimportant when we take $b$ infinitesimally close to 1 , a procedure with many advantages. ${ }^{23-25}$ After $m \mathrm{RG}$ iterations the slab is reduced to a double layer $(z=2)$ and renormalization then proceeds in a way that exactly solves the double chain $(d=2)$, as outlined in Appendix $\mathbf{B} 1$.

In the case $h_{1}=h_{2}=0$, one obtains

$$
\begin{aligned}
-F / n^{d-1}-\left[\left(b^{m}-1\right) d+1\right] K_{B}-2(d-1) K_{S}= & \sum_{k=0}^{m-1}\left(b^{1-d}\right)^{k+1}\left\{\left[d\left(b^{m-k-1}-1\right)+1\right] g_{B}^{(k)}+2(d-1) g_{S}^{(k)}\right\} \\
& +\sum_{k=m}^{\infty}\left(b^{1-d}\right)^{k+1}(d-1) g_{D}^{(k)}
\end{aligned}
$$

The contribution of the dangling spins is omitted since it vanishes for $b \rightarrow 1$. Furthermore,

$$
g_{S}^{(k)}=\ln \left\{\frac{1}{2}\left[\left(1+\tilde{\rho}_{S}^{(k)}\right)^{b}+\left(1-\tilde{\rho}_{S}^{(k)}\right)^{b}\right]\right\},
$$

where

$$
\tilde{\rho}_{S}^{(k)} \equiv\left(\rho_{B}^{(k)}\right)^{\left(b^{d-1}-b^{d-2}\right) / 2}\left(\rho_{S}^{(k)}\right)^{b^{d-2}},
$$

with

$$
\rho_{S}^{(k)} \equiv \exp \left(-2 K_{S}^{(k)}\right)
$$

and the recursion relation for $\rho_{S}$ is as in (4.3), replacing $\rho_{B}$ by $\rho_{S}$.

The first sum in (4.4) corresponds to the first $m$ RG iterations which reduce the slab to two layers of $\left(n / b^{m}\right)^{d-1}$ sites each, with intralayer coupling $K_{S}^{(m)}$ and interlayer coupling $K_{B}^{(m)}$. Denote a top-layer spin by $s_{i}$ and its nearest-neighbor bottom-layer spin by $t_{i}$. As explained in Appendix B 1, new Boltzmann weights $x\left(s_{i}, t_{i} ; s_{j}, t_{j}\right)$ are defined for pairs $\left(s_{i}, t_{i}\right)$ and $\left(s_{j}, t_{j}\right)$, where $s_{i}$ and $s_{j}$ are nearest neighbors. Every further RG iteration contributes a term proportional to $g_{D}$ to the free energy, where

$$
g_{D}=\ln _{\left\{s_{i}, t_{i} ; s_{j}, t_{j}\right\}} x\left(s_{i}, t_{i} ; s_{j}, t_{j}\right),
$$

ensuring proper normalization of the Boltzmann weights.

The surface free energy per column now follows from combining (4.1) and (4.4),

$$
\begin{aligned}
f_{S}= & \sum_{k=0}^{m-1}\left(b^{1-d}\right)^{k+1}(d-1)\left(g_{B}^{(k)}-2 g_{S}^{(k)}\right) \\
& +\sum_{k=m}^{\infty}\left(b^{1-d}\right)^{k+1}\left[d b^{m-k-1} g_{B}^{(k)}-(d-1) g_{D}^{(k)}\right],
\end{aligned}
$$

where we have replaced $z$ in (1.2) by $z-1$, a more con- venient choice of thickness of the reference bulk system in this case. Furthermore, terms that do not depend on $z$ or that vanish for $b \rightarrow 1$ have been omitted.

It is now evident that, at bulk criticality, $f_{S}$ depends on $z$ through $\Delta / z^{d-1}$ for $z \rightarrow \infty$. Take $K_{S}=K_{B}=K_{c}^{(d)}$, for example. The bulk coupling is then at the critical fixed point $K_{B}^{*}=K_{c}^{(d)}$, and $K_{S}$ renormalizes to $K_{0}^{*}$ associated with the fixed point of the ordinary transition. The difference $g_{B}-2 g_{S}$ in the first sum of (4.4) converges to its fixed-point value $g_{B}^{*}-2 g_{0}^{*}$ exponentially fast. Therefore, in the limit $z \rightarrow \infty(m \rightarrow \infty)$, a contribution

$$
-\frac{\left(b^{1-d}\right)^{m}}{b^{d-1}-1}(d-1)\left(g_{B}^{*}-2 g_{0}^{*}\right)
$$

results. Analogously, the second sum of (4.4) contributes

$$
\frac{\left(b^{1-d}\right)^{m}}{b^{d}-1} d g_{B}^{*}-(d-1)\left(b^{1-d}\right)^{m} \sum_{k=0}^{\infty}\left(b^{1-d}\right)^{k+1} g_{D, 0}^{(m+k)} \text {. }
$$

Taking the limit of an infinitesimal rescaling $b=1+\epsilon$, $0<\epsilon<1$, the previous two results add up to

$z^{1-d}\left[2 \hat{g}_{0}^{*}-(d-1) \int_{0}^{\infty} d l e^{(1-d) l} \hat{g}_{D, 0}(l)\right] \equiv z^{1-d} \Delta_{O, O}$,

where $\hat{g} \equiv \lim _{\epsilon \rightarrow 0}(g / \epsilon)$, and $g_{D, 0}(l=0) \equiv g_{D}\left(K_{B}^{*}, K_{O}^{*}\right)$.

In the following, we consider the case $h_{1} \neq 0=h_{2}$. To obtain the amplitude $\Delta_{O,+}$, for example, the appropriate RG fixed point is $K_{B}=K_{c}^{(d)}, K_{S}=K_{O}^{*}$, and $h_{1}=+\infty$. The spins in the top layer are then frozen $\left(s_{i}=1\right)$ and once the Boltzmann weights have been normalized this layer no longer contributes to the free energy. After bond moving, the spin chains that terminate on one end at the top layer are decimated just like spin chains further down in the slab. The part of the surface free energy that yields $\Delta_{O,+}$ is then given by

$$
f_{S}\left(h_{1}=+\infty\right)=\sum_{k=0}^{m-1}\left(b^{1-d}\right)^{k+1}(d-1)\left(g_{B}^{(k)}-g_{S}^{(k)}\right)+\sum_{k=m}^{\infty}\left(b^{1-d}\right)^{k+1}\left[d b^{m-k-1} g_{B}^{(k)}-(d-1) g_{D}^{(k)}(\infty)\right],
$$

where $g_{D}(\infty)$ is obtained as explained in Appendix B 2 .

Result (4.9) differs from (4.7): contributions $g_{S}^{(k)}$ to the free energy arise only at surface layers with zero boundary field; after $m$ RG iterations the double layer presently $\left(h_{1}=+\infty\right)$ consists of a $(d-1)$-dimensional single layer of spins on which a field of strength $K_{B}=K_{c}^{(d)}$ acts.

Next consider $h_{1} h_{2}>0$. To obtain $\Delta_{+,+}$the appropriate fixed point is $K_{B}=K_{c}^{(d)}, h_{1}=h_{2}= \pm \infty$. The value of 
$K_{S}(>-\infty)$ is irrelevant since the surface spins are all +1 or all -1 . In the $m$ th RG iteration, spin chains are decimated which connect top to bottom layer and have both end-point spins +1 or both -1 . The contribution to the free energy is exactly the same as if both end-point spins were free. However, this iteration now terminates the renormalization of the slab. The part of the surface free energy that yields $\Delta_{+,+}$is

$$
\begin{aligned}
f_{S}\left(h_{1}=h_{2}= \pm \infty\right)= & \sum_{k=0}^{m-1}\left(b^{1-d}\right)^{k+1}(d-1) g_{B}^{(k)} \\
& +\sum_{k=m}^{\infty}\left(b^{1-d}\right)^{k+1} d b^{m-k-1} g_{B}^{(k)} .
\end{aligned}
$$

It follows that $\Delta_{+,+}=0$ in the limit $b \rightarrow 1$. This lack of interaction appears to be a peculiarity of the MigdalKadanoff approximation.

In the case $h_{1} \dot{h}_{2}<0$, to extract $\Delta_{+,-}$, the appropriate fixed point is $K_{B}=K_{c}^{(d)}, h_{1}=-h_{2}= \pm \infty$. Unlike in the previous case $h_{1} h_{2}>0$, the $m$ th RG iteration now decimates spin chains with opposite end-point spins. This results in a different contribution to the free energy given by

$$
\begin{aligned}
g_{B}^{(m-1)}(+-) & =\ln \left\{\frac{1}{2}\left[\left(1+\widetilde{\rho}_{B}^{(m-1)}\right)^{b}-\left(1-\tilde{\rho}_{B}^{(m-1)}\right)^{b}\right]\right\} \\
& =g_{B}^{(m-1)}+\ln \rho_{B}^{(m)} .
\end{aligned}
$$

It follows that

$$
\begin{aligned}
f_{S}\left(h_{1}=-h_{2}= \pm \infty\right) & =\sum_{k=0}^{m-2}\left(b^{1-d}\right)^{k+1}(d-1) g_{B}^{(k)}+b^{(1-d) m}\left[d g_{B}^{(m-1)}-g_{B}^{(m-1)}(+-)\right]+\sum_{k=m}^{\infty}\left(b^{1-d}\right)^{k+1} d b^{m-k-1} g_{B}^{(k)} \\
& =f_{S}\left(h_{1}=h_{2}= \pm \infty\right)-\left(b^{1-d}\right)^{m} \ln \rho_{B}^{(m)}
\end{aligned}
$$

Therefore, in this approximation,

$$
\Delta_{+,-}=\Delta_{+,+}+2 K_{c}^{(d)} \text {. }
$$

Up to now the surface coupling $K_{S}$ has been either renormalizing toward $K_{O}^{*}$ (ordinary transition, $h_{i}=0$ ), or irrelevant (in the presence of a surface field). In the following, we examine the cases $h_{1}=h_{2}=0$ and $h_{1}=0 \neq h_{2}$, which involve the fixed points of the surface-bulk and extraordinary transitions.

Consider $h_{1}=h_{2}=0$. The appropriate fixed point for computing $\Delta_{E, E}$ is $K_{B}=K_{c}^{(d)}, K_{S}=K_{E}^{*}=+\infty$ on both surface layers. Nearest-neighbor spins on the same surface are now fixed to the same value. By calculating analytically the free energy of the double layer to which the slab reduces after $m$ RG iterations, one sees that in the thermodynamic limit $(n \rightarrow \infty)$ the only surviving configurations have the same spin orientation on both surface layers. Therefore, $\Delta_{E, E}=\Delta_{+,+}$. We expect this result to be generally valid: if both surfaces are at the extraordinary transition, they order in the same direction in equilibrium. This is physically indistinguishable from the case where the surfaces order due to fields in the same direction.

Next we turn to $\Delta_{\mathrm{SB}, \mathrm{SB}}$, corresponding to the fixed point $K_{B}=K_{c}^{(d)}, K_{S}=K_{\mathrm{SB}}^{*}$ on both surfaces $\left(h_{1}=h_{2}=0\right)$. This amplitude is computed similarly to $\Delta_{O, O}$, replacing $K_{O}^{*}$ by $K_{\mathrm{SB}}^{*}$.

Also cases where top and bottom layers have different surface couplings are interesting for $h_{1}=h_{2}=0$. Consider first $\Delta_{O, E}$, associated with the fixed point $K_{B}=K_{c}^{(d)}$, $K_{S}=K_{O}^{*}$ (top), $K_{S}=K_{E}^{*}$ (bottom). Since one surface is ordered, we expect the equality $\Delta_{O, E}=\Delta_{O,+}$ to hold in general. Two more fixed points exist: $K_{S}=K_{\mathrm{SB}}^{*}$ (top) with $K_{S}=K_{O}^{*}$ or $K_{S}=K_{E}^{*}$ (bottom), at which two new independent amplitudes are computed: $\Delta_{O, \mathrm{SB}}$ and $\Delta_{\mathrm{SB}, E}$.

In the following we take $h_{1}=0 \neq h_{2}$. Consider then $K_{S}=K_{\mathrm{SB}}^{*}$ or $K_{S}=K_{E}^{*}$ on the top layer. Following the same reasoning as above, we expect that, in general, $\Delta_{\mathrm{SB},+}=\Delta_{\mathrm{SB}, E}$ and $\Delta_{E,+}=\Delta_{+,+}$.

In the remainder of this section, we discuss the numerical results and test their accuracy. For completeness, we show in Table II the numerical values of bulk and surface fixed-point couplings. In Table I (see Introduction) we have presented the finite-size interaction amplitudes. Exact results are available in $\mathrm{two}^{2,5}$ and four ${ }^{3}$ dimensions. They are included in the table, in parentheses. The Migdal-Kadanoff $R G$ results agree surprisingly well with the exact numbers, suggesting that we may trust the RG results in $d=3$, at least in order of magnitude.

One direct check of the accuracy in $d=3$ lies in the computation by Migdal-Kadanoff RG of the finite-size amplitude $Y$ of the excess free energy per column of a periodic slab, i.e., with periodic boundary conditions also in the direction perpendicular to the layers. Besides comparing the result in $d=2$ with the exact value, one can check the prediction in $d=3$ against a recent Monte Carlo estimate by Mon and Nightingale, ${ }^{6}$ who computed

$$
\Delta(0)=\Delta_{o, o}-Y,
$$

where $Y$ is defined as the amplitude of the $z^{1-d}$ contribution to the free energy per column of an infinite slab of $z$ layers with periodic boundary conditions in all directions.

TABLE II. Migdal-Kadanoff renormalization-group fixed points $K_{B}^{*}$ for the bulk coupling $K_{B}$ at criticality, and $K_{O}^{*}$ and $K_{\mathrm{SB}}^{*}$ for the surface coupling $K_{S}$ at the ordinary and surfacebulk transitions, respectively in 2,3 , and 4 dimensions.

\begin{tabular}{cccc}
\hline \hline$d$ & $K_{B}^{*}=K_{c}^{(d)}$ & $K_{O}^{*}$ & $K_{\mathrm{SB}}^{*}$ \\
\hline 2 & 0.4407 & 0.0915 & \\
3 & 0.1398 & 0.0266 & 0.3393 \\
4 & 0.0501 & 0.0094 & 0.1102 \\
\hline \hline
\end{tabular}


TABLE III. Migdal-Kadanoff renormalization-group estimates for the finite-size amplitudes $\Delta(0)=\Delta_{O, o}-Y$, and $Y$ in various dimensions $d$. Numbers in parentheses are exact results or Monte Carlo estimates.

\begin{tabular}{lcc}
\hline \hline$d$ & $\Delta(0)$ & $Y$ \\
\hline 2 & 0.086 & -0.141 \\
& $(0.197)^{\mathrm{a}}$ & $(-0.262)^{\mathrm{a}}$ \\
3 & 0.067 & -0.082 \\
& $(0.1)^{\mathrm{b}}$ & \\
4 & 0.111 & -0.118 \\
\hline \hline
\end{tabular}

${ }^{\text {aReference } 2 \text {, exact. }}$

${ }^{\text {b}}$ Reference 6, Monte Carlo.

To compute $Y$, we work with a periodic slab of $z$ layers and $2 b^{m} n^{d-1}$ sites. We choose $z=2 b^{m}$ so that $m \mathrm{RG}$ iterations reduce the slab to a double layer. The excess free energy per column is defined as

$$
f^{(p)}=\lim _{n \rightarrow \infty}\left(F^{(p)}-z n^{d-1} f_{B}\right) / n^{d-1},
$$

where $F^{(p)}$ is the total free energy of a slab that is periodic in all directions. In analogy with the calculation that led to (4.7), we obtain

$$
f^{(p)}=\sum_{k=m}^{\infty}\left(b^{1-d}\right)^{k+1}\left[2 d b^{m-k-1} g_{B}^{(k)}-(d-1) g_{D}^{(k)}\right] \text {. }
$$

Note that the renormalization of the double layer is now initialized with $K_{B}^{(m)}=2 K_{B}^{*}$ (periodic boundary conditions) and $K_{S}^{(m)}=K_{B}^{*}$. At the fixed point corresponding to bulk criticality, we obviously obtain $f^{(p)}=Y z^{1-d}$. The numerical results for $Y$ and $\Delta(0)$ are shown in Table III and compared with exact $(d=2)$ (last of Ref. 2) and Monte Carlo $(d=3)$ (Ref. 6) results. The agreement is satisfactory. In particular, the Migdal-Kadanoff $R G$ result for $\Delta(0)$ is of the same order of magnitude as that from the Monte Carlo simulations.

\section{ACKNOWLEDGMENTS}

Useful conversations with Professor Nihat Berker, Professor Michael Schick, and Professor Fred Wu, and helpful remarks by Dr. S. Dietrich and Dr. S. Leibler, are kindly acknowledged. One of us (M.P.N.) thanks the "Instituut voor Theoretische Fysica" of the "Katholieke Universiteit Leuven," where part of this work was done, for its hospitality. Another of us (J.O.I.) thanks the Belgian National Fund for Scientific Research for financial aid. The research at the Massachusetts Institute of Technology was funded by the U.S. Army Research Office under Contract No. DAAG29-85-K0058 and that at the University of Rhode Island under National Science Foundation Contract No. DMR-84-06186.

\section{APPENDIX A: EXACT RESULTS}

\section{Free-energy calculation}

To solve the triangular Ising model [see Eq. (2.1)] with cylindrical boundary conditions, we employ the method as used to solve the Ising model on a finite, simple quadratic lattice with a boundary field (see Ref. 14, Chap. VI). Here we sketch the derivation. ${ }^{26}$

The counting lattice used is illustrated in Figs. 8.6 (p. 190), and 8.8 (p.191) of Ref. 14. The partition function of a lattice of $m$ columns (free top and bottom) and $n$ rows (periodic) reads

$$
Z_{m n}=\left(2 \cosh K_{1}\right)^{m n}\left(\cosh K_{2} \cosh K_{3}\right)^{m(n-1)}(\operatorname{det} A)^{1 / 2},
$$

with

$$
\operatorname{det} A=\prod_{\theta} \operatorname{det} B(\theta)
$$

where the product is over $\theta=\pi(2 p-1) / m, p=1$, $2, \ldots, m$. Here, $B(\theta)$ is a tridiagonal, $n \times n$ block matrix with $6 \times 6$ blocks:

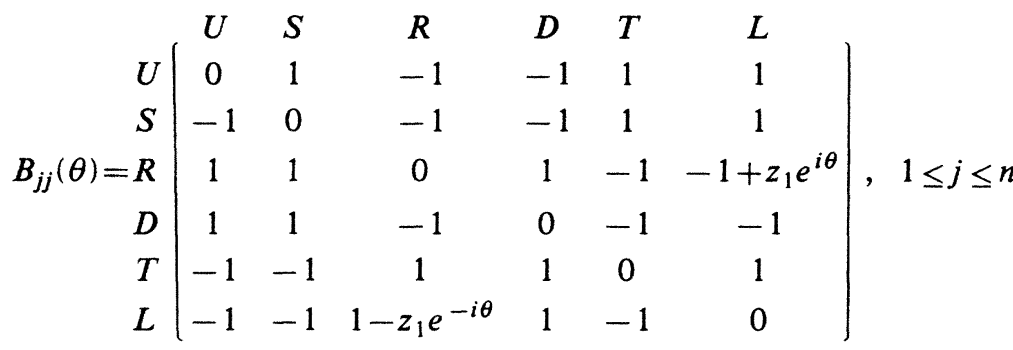

$$
\begin{aligned}
& B_{j, j+1}(\theta)=-B_{j+1, j}(\theta)=\begin{array}{c}
U \\
S \\
S \\
D \\
T \\
T \\
L
\end{array}\left(\begin{array}{cccccc}
U & S & R & D & T & L \\
0 & 0 & 0 & z_{2} & 0 & 0 \\
0 & 0 & 0 & 0 & -z_{3} e^{i \theta} & 0 \\
0 & 0 & 0 & 0 & 0 & 0 \\
0 & 0 & 0 & 0 & 0 & 0 \\
0 & 0 & 0 & 0 & 0 & 0 \\
0 & 0 & 0 & 0 & 0 & 0
\end{array}\right), \quad 1 \leq j<n
\end{aligned}
$$


where $z_{i}=\tanh K_{i}$. The rows and columns labeled $R$ and $L$ are eliminated as follows. A matrix $B^{\prime}(\theta)$ is obtained from $B(\theta)$ with $B^{\prime}(\theta)=T(\theta) B(\theta)$, where $T(\theta)$ is block diagonal with blocks

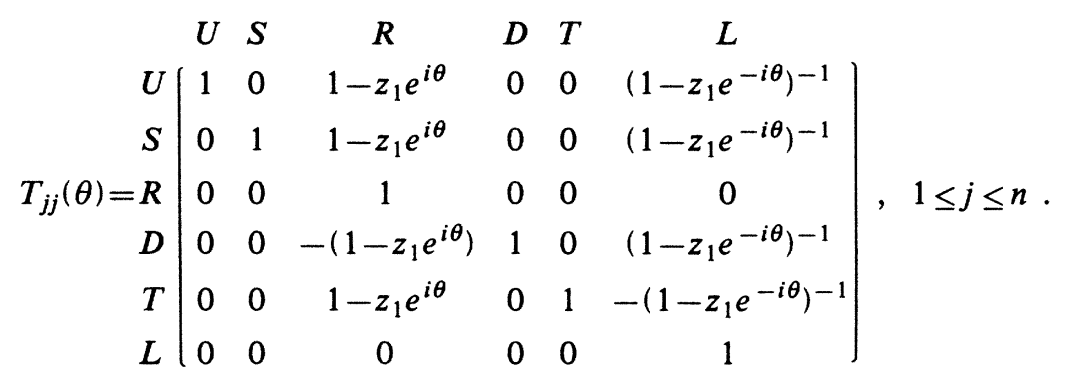

Since $\operatorname{det} T(\theta)=1$, one has $\operatorname{det} B(\theta)=\operatorname{det} B^{\prime}(\theta)$. Expanding $\operatorname{det} B^{\prime}(\theta)$ in the $R$ and $L$ columns yields

$$
\operatorname{det} A=\prod_{\theta}\left[\left|1-z_{1} e^{i \theta}\right|^{2 n} \operatorname{det} \bar{B}(\theta)\right],
$$

where $\bar{B}(\theta)$ again is a tridiagonal, $n \times n$ block matrix. This time the blocks are $4 \times 4$,

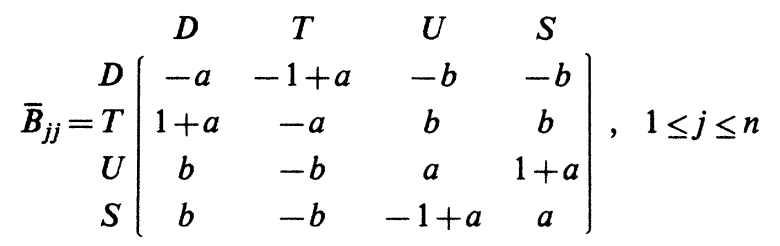

$$
\begin{aligned}
& \left.\bar{B}_{j, j+1}=-\bar{B}_{j+1, j}=\begin{array}{r|cccc}
D & T & U & S \\
D & 0 & 0 & 0 & 0 \\
U & 0 & 0 & 0 \\
S & 0 & 0 & 0 \\
z_{2} & 0 & -z_{3} e^{i \theta} & 0 & 0
\end{array}\right), \quad 1 \leq j<n
\end{aligned}
$$

where

$$
\begin{aligned}
& a=2 i z_{1}\left|1-z_{1} e^{i \theta}\right|^{-2} \sin \theta, \\
& b=\left(1-z_{1}^{2}\right)\left|1-z_{1} e^{i \theta}\right|^{-2} .
\end{aligned}
$$

Denote $\operatorname{det} \bar{B}(\theta)$ by $P_{n}$, and by $Q_{n}$ the determinant of the $(4 n-1) \times(4 n-1)$ matrix obtained by removing the first row and column of the matrix $\bar{B}(\theta)$. It can be shown that $P_{n}$ and $Q_{n}$ satisfy the following recursion relations,

$$
\left[\begin{array}{c}
P_{n} \\
\sqrt{|E / C|} Q_{n}
\end{array}\right)=\left(\begin{array}{cc}
A & i \sqrt{|E C|} \\
-i \sqrt{|E C|} & F
\end{array}\right)\left(\begin{array}{c}
P_{n-1} \\
\sqrt{|E / C|} Q_{n-1}
\end{array}\right),
$$

with

$$
\left(\begin{array}{l}
P_{1} \\
Q_{1}
\end{array}\right)=\left(\begin{array}{c}
1 \\
-a
\end{array}\right)
$$

and where

$$
\begin{aligned}
& A=\left(\alpha^{2}+\eta^{2}+2 \alpha \eta \cos \theta\right) /\left|1-z_{1} e^{i \theta}\right|^{2}, \\
& i E=(2 i \gamma \beta \sin \theta) /\left|1-z_{1} e^{i \theta}\right|^{2}, \\
& i C=(2 i \alpha \eta \sin \theta) /\left|1-z_{1} e^{i \theta}\right|^{2}, \\
& F=\left(\gamma^{2}+\beta^{2}+2 \gamma \beta \cos \theta\right) /\left|1-z_{1} e^{i \theta}\right|^{2}, \\
& \alpha=z_{1}+z_{2} z_{3}, \quad \beta=z_{2}+z_{3} z_{1}, \\
& \gamma=z_{3}+z_{1} z_{2}, \quad \eta=-\left(z_{1} z_{2} z_{3}+1\right),
\end{aligned}
$$

from Eq. (A11) it follows that

$$
P_{n}=p_{+} \lambda_{+}^{n-1}+p_{-} \lambda_{-}^{n-1}
$$


where

$$
\begin{aligned}
& P_{+}=\frac{|E C|+\bar{a}|E|\left(A-\lambda_{+}\right)}{|E C|+\left(A-\lambda_{+}\right)^{2}}, \\
& P_{-}=\frac{\left(A-\lambda_{+}\right)^{2}-\bar{a}|E|\left(A-\lambda_{+}\right)}{|E C|+\left(A-\lambda_{+}\right)^{2}} \text {, } \\
& \lambda_{ \pm}=\frac{A+F \pm\left[(A-F)^{2}+4|E C|\right]^{1 / 2}}{2} \\
& =\frac{1}{2}\left(1+z_{1}^{2}\right)\left(1+z_{2}^{2}\right)\left(1+z_{3}^{2}\right)-4 z_{1} z_{2} z_{3}-\cos (\theta) z_{1}\left(1-z_{2}^{2}\right)\left(1-z_{3}^{2}\right) \pm \frac{1}{2} \Delta,
\end{aligned}
$$

where

$$
\Delta^{2}=\left(1-z_{2}^{2}\right)^{2}\left(1-z_{3}^{2}\right)^{2}\left(1+z_{1}^{2}+2 z_{1} \cos \theta\right)^{2}+16\left(z_{1}-z_{2} z_{3}\right)\left(z_{2}-z_{3} z_{1}\right)\left(z_{3}-z_{1} z_{2}\right)\left(z_{1} z_{2} z_{3}-1\right) .
$$

with $\left|\lambda_{+}\right| \geq\left|\lambda_{-}\right|$.

From Eqs. (A1), (A6), and (A13), it follows that $f=-\ln Z_{n} / m n$, the free energy per site, is given by

$$
f=-\ln \left(2 \cosh K_{1} \cosh K_{2} \cosh K_{3}\right)+\frac{1}{n}\left(\cosh K_{2} \cosh K_{3}\right)-\frac{1}{2 m n} \sum_{\theta} \ln \left[\left|1-z_{1} e^{i \theta}\right|^{2 n}\left(p_{+} \lambda_{+}^{n-1}+p_{-} \lambda_{-}^{n-1}\right)\right] .
$$

From this expression the finite-size interaction for $m \rightarrow \infty$ is found

$$
\Delta_{0,0}=-\lim _{n \rightarrow \infty} n^{2} \int_{0}^{2 \pi} \frac{d \theta}{4 \pi} \ln \left[1+\left(\frac{\lambda_{-}}{\lambda_{+}}\right)^{n}\left(\frac{p_{-}}{p_{+}}\right)\right] .
$$

(A16)

Dominant contributions to the integral come from the neighborhoods of $\theta=0$ and $\theta=2 \pi$, where

$$
\begin{aligned}
\frac{\lambda_{-}}{\lambda_{+}} & =1-\frac{2}{\eta} \theta+O\left(\theta^{2}\right) \\
& =1-\frac{2}{\eta}(2 \pi-\theta)+O\left((2 \pi-\theta)^{2}\right),
\end{aligned}
$$

with

$$
\begin{aligned}
\eta & =\frac{\left(1+z_{1}^{2}\right)\left(1+z_{2}^{2}\right)\left(1+z_{3}^{2}\right)+8 z_{1} z_{2} z_{3}-2 z_{1}\left(1-z_{2}^{2}\right)\left(1-z_{3}^{2}\right)}{4\left[\left(z_{1}+z_{2} z_{3}\right)\left(z_{2}+z_{3} z_{1}\right)\left(z_{3}+z_{1} z_{2}\right)\left(z_{1} z_{2} z_{3}+1\right)\right]^{1 / 2}} \\
& =\frac{C_{2}^{2}}{S_{1}+S_{2}},
\end{aligned}
$$

where we used $z_{i}=\left(C_{i}-1 / S_{i}\right)$ and $S_{1} S_{2}+S_{2} S_{3}$ $+S_{3} S_{1}=1$. One finds

$\Delta_{O, O}=-\frac{1}{2 \pi} \int_{0}^{\infty} d \theta \ln \left(1+e^{-2 \theta / \eta}\right)=-\frac{\pi}{48} \eta$,

which is Eq. (2.4).

\section{Correlation-length calculation}

We need to find the correlation lengths along the three directions of the bonds of the triangular lattice. We use the following expression ${ }^{27}$ for $g_{k}^{(i)}$, the spin-spin correlation function of two spins $k$ lattice sites apart along the direction of the $K_{i}$ bonds:

$$
g_{k}^{(i)}=\operatorname{det}\left(\begin{array}{cccc}
a_{0}^{(i)} & a_{1}^{(i)} & \cdots & a_{k-1}^{(i)} \\
a_{-1}^{(i)} & a_{0}^{(i)} & \cdots & a_{k-2}^{(i)} \\
\vdots & \vdots & \vdots & \vdots \\
a_{1-k}^{(i)} & a_{2-k}^{(i)} & \cdots & a_{0}^{(i)}
\end{array}\right)
$$

where

$$
a_{n}^{(i)}=-\frac{1}{2 \pi} \int_{-\pi}^{\pi} e^{-i n \omega} \frac{a^{(i)}-b^{(i)} e^{i \omega}-c^{(i)} e^{-i \omega}}{a^{(i)}-b^{(i)} e^{-i \omega}-c^{(i)} e^{i \omega}} d \omega
$$

with

$$
\begin{aligned}
& a^{(i)}=2 z_{i}\left(1+z_{j}^{2}\right)\left(1+z_{k}^{2}\right)+4 z_{j} z_{k}\left(1+z_{i}^{2}\right), \\
& b^{(i)}=z_{i}^{2}\left(1-z_{j}^{2}\right)\left(1-z_{k}^{2}\right), \\
& c^{(i)}=\left(1-z_{j}^{2}\right)\left(1-z_{k}^{2}\right)
\end{aligned}
$$

$$
(i, j, k=1,2,3 \text { or cyclic permutations). }
$$

One finds $a_{n}^{(i)} \sim e^{-n / \xi_{i}}$, where $\xi_{i}$ can be expressed in terms of the closest singularity of the integrand in (A20) to the real axis:

$$
\xi_{i}^{-1}=\ln \gamma_{+}^{(i)}
$$

where

$$
\gamma_{+}^{(i)}=\frac{a^{(i)}+\left[\left(a^{(i)}\right)^{2}-4 b^{(i)} c^{(i)}\right]^{1 / 2}}{2 c^{(i)}}
$$

From Eq. (A22) one can derive Eqs. (2.6) without further assumptions.

We conjecture that $\xi_{i}$ defined in this way indeed is the correlation length. Indeed, for the rectangular and the isotropic triangular lattices this gives the correct results. 


\section{APPENDIX B: MIGDAL-KADANOFF RG COMPUTATIONS}

\section{Renormalization of a double layer}

This appendix is devoted to the Migdal-Kadanoff RG computation of the free energy of a double-layer. The method which we employ is analogous to that of Ref. 28.
The intralayer nearest-neighbor coupling is $K_{S}$ and that between the layers is $K_{B}$. Denote a top-layer spin by $s_{i}$ and its nearest neighbor in the bottom layer by $t_{i}$. Consider two pairs, $\left(s_{i}, t_{i}\right)$ and $\left(s_{j}, t_{j}\right)$, where $s_{i}$ and $s_{j}$ are nearest neighbors. The associated Boltzmann weight whose form is invariant under the bond-moving RG approximation is given by

$$
x\left(s_{i}, t_{i} ; s_{j}, t_{j}\right)=\exp \left[\frac{K_{B}}{2(d-1)}\left(s_{i} t_{i}+s_{j} t_{j}-2\right)+K_{S}\left(s_{i} s_{j}+t_{i} t_{j}-2\right)+K_{D}\left(s_{i} t_{j}+s_{j} t_{i}-2\right)+K_{F}\left(s_{i} s_{j} t_{i} t_{j}-1\right)\right],
$$

where the couplings $K_{D}$ and $K_{F}$ represent next-nearestneighbor (diagonal) and four-spin interactions, respectively, which are generated by the RG transformation. Note that the weights are normalized such that $x(1,1 ; 1,1)=1$. For simplicity only a single surface interaction is assumed here.

The $4 \times 4$ transfer matrix $X$ between $i$ and $j$ is now constructed as follows: Let the indices $1-4$ correspond to the four states of spins $s$ and $t$, in the order,+++- , -- , and -+ . We then have

$$
X=\left(\begin{array}{llll}
1 & \beta & \delta & \beta \\
\beta & \gamma & \beta & \epsilon \\
\delta & \beta & 1 & \beta \\
\beta & \epsilon & \beta & \gamma
\end{array}\right),
$$

where, e.g., $X_{12}=X(1,1 ; 1,-1)=\beta$.

The "bond-moving" step in the RG iteration amounts to the replacement $X \rightarrow \widetilde{X}$, where

$$
\widetilde{X}_{k l} \equiv\left(X_{k l}\right)^{b^{d-2}}
$$

since the layer has dimension $d-1$ (the length rescaling is b).

The next operation is the decimation step, which yields the renormalized transfer matrix

$$
X^{\prime}=\widetilde{X}^{b} .
$$

First, we compute $U^{-1} \widetilde{X}^{b} U$, which equals $\left(U^{-1} \tilde{X} U\right)^{b}$, where

$$
U=\frac{1}{\sqrt{2}}\left(\begin{array}{ll}
1 & 0 \\
0 & 1
\end{array}\right) \text { and } \mathbf{1} \equiv\left(\begin{array}{ll}
1 & 0 \\
0 & 1
\end{array}\right)
$$

We finally obtain

$$
X^{\prime}=U\left(\begin{array}{cccc}
a^{\prime} & d^{\prime} & 0 & 0 \\
d^{\prime} & c^{\prime} & 0 & 0 \\
0 & 0 & \lambda_{1}^{\prime} & 0 \\
0 & 0 & 0 & \lambda_{2}^{\prime}
\end{array}\right) U^{-1}
$$

where

$$
\begin{aligned}
& a^{\prime}=u_{1} \lambda_{+}^{b} u_{1}+v_{1} \lambda_{-}^{b} v_{1}, \\
& c^{\prime}=u_{2} \lambda_{+}^{b} u_{2}+v_{2} \lambda_{-}^{b} v_{2}, \\
& d^{\prime}=u_{1} \lambda_{+}^{b} u_{2}+v_{1} \lambda_{-}^{b} v_{2},
\end{aligned}
$$

with $\lambda_{+}$and $\lambda_{-}$the eigenvalues, and $u$ and $v$ the respective eigenvectors of the $2 \times 2$ matrix

$$
A=\left[\begin{array}{cc}
1+\widetilde{\delta} & 2 \widetilde{\beta} \\
2 \widetilde{\beta} & \widetilde{\gamma}+\widetilde{\epsilon}
\end{array}\right) .
$$

Furthermore,

$$
\lambda_{1}^{\prime}=(1-\widetilde{\delta})^{b}
$$

and

$$
\lambda_{2}^{\prime}=(\widetilde{\gamma}-\widetilde{\epsilon})^{b} .
$$

The subsequent normalization of $X^{\prime}$ then determines the contribution to the free energy $g_{D}$ via (4.6), which takes the form

$$
g_{D}=\ln X_{11}^{\prime}=\ln \left[\frac{1}{2}\left(a^{\prime}+\lambda_{1}^{\prime}\right)\right]
$$

because $X_{11}$ is always the largest element of $X$ in the actual computations. We remark that for $d=2$ the RG procedure yields the exact free energy of the double chain.

\section{B. Renormalization of a single layer in a field}

In this section we treat the renormalization of a single layer of spins in $d-1$ dimensions, with nearest-neighbor coupling $K_{S}$ and magnetic field of strength $h=K_{B}$.

The normalized Boltzmann weight between nearest neighbors $s_{i}$ and $s_{j}$ is then given by

$$
x\left(s_{i}, s_{j}\right)=\exp \left(K_{S}\left(s_{i} s_{j}-1\right)+\frac{K_{B}}{d-1} \frac{s_{i}+s_{j}-2}{2}\right),
$$

where we have distributed the magnetic field terms over the nearest-neighbor pairs.

The associated $2 \times 2$ transfer matrix then reads

$$
X=\left(\begin{array}{ll}
1 & \beta \\
\beta & \gamma
\end{array}\right),
$$

with $\beta=x(1,-1)$ and $\gamma=x(-1,-1)$.

The "bond-moving" step of the RG calculation is again defined by (B3), and the decimation step by (B4).

We obtain

$$
X^{\prime}=\left(\begin{array}{ll}
a^{\prime} & d^{\prime} \\
d^{\prime} & c^{\prime}
\end{array}\right)
$$

where $a^{\prime}, d^{\prime}$, and $c^{\prime}$ are defined by (B6), with $\lambda_{+}$and $\lambda_{-}$ the eigenvalues and $u$ and $v$ the respective eigenvectors of $X$.

In analogy with (B9), we now have

$$
g_{D}(\infty)=\ln X_{11}^{\prime}=\ln a^{\prime} .
$$


*Current address: Laboratorium voor Technische Natuurkunde, Technische Hogeschool Delft, Delft, The Netherlands.

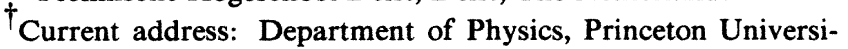
ty, Princeton, NJ 08544.

${ }^{1}$ M. E. Fisher and P.-G. de Gennes, C. R. Acad. Sci. Paris Ser. B 287, 207 (1978).

${ }^{2}$ H. Au-Yang and M. E. Fisher, Phys. Rev. B 11, 3469 (1975); 21, 3956 (1980); M. E. Fisher and H. Au-Yang, Physica (Utrecht) 101A, 255 (1980); A. E. Ferdinand and M. E. Fisher, Phys. Rev. 185, 832 (1969).

${ }^{3}$ K. Symanzik, Nucl. Phys. B190, [FS3], 1 (1981).

${ }^{4}$ R. Lipowsky and U. Seifert, Phys. Rev. B 31, 4701 (1985).

${ }^{5}$ M. P. Nightingale and J. O. Indekeu, Phys. Rev. Lett. 54, 1824 (1985).

${ }^{6}$ K. K. Mon and M. P. Nightingale, Phys. Rev. B 31, 6137 (1985).

${ }^{7}$ V. Privman and M. E. Fisher, Phys. Rev. B 30, 322 (1984).

${ }^{8}$ S. Singh and R. K. Pathria, Phys. Rev. Lett. 55, 347 (1985).

${ }^{9} \delta f_{\text {sing }}=\delta f-\delta f_{\text {reg }}$, where size dependence of the regular part $\delta f_{\text {reg }}$ in Eq. (1.4) is defined, in principle, via the size dependence of the functions $g$ as in Eq. (4.7). As long as there is no $z^{-(d-1)}$ dependence in the $g$ (a behavior hard to reconcile with locality concepts of renormalization-group theory), the finite-size amplitudes of $\delta f$ and $\delta f_{\text {sing }}$ are identical. Also see Ref. 7 and H. W. J. Blöte and M. P. Nightingale, Physica (Utrecht) 112A, 405 (1982).

${ }^{10}$ See, for example, H. W. Diehl, J. Appl. Phys. 53, 7914 (1982); T. W. Burkhardt and E. Eisenriegler, Phys. Rev. B 24, 1236 (1981), and references therein.

${ }^{11}$ H. W. J. Blöte, J. L. Cardy, and M. P. Nightingale, Phys. Rev. Lett. 56, 742 (1986); I. Affleck, ibid. 56, 746 (1986). Note that in the case of opposite boundary conditions corresponding to $\Delta_{+,-}$, neither exact nor conformal invariance results have yet been obtained, which could prove or disprove the conjecture $\Delta_{+,-}=2^{d} \Delta_{0,+}$ in $d=2$.

${ }^{12}$ M. E. Fisher, Proc. Nobel Symp. 24, 16 (1973); also see second of Ref. 2.

${ }^{13}$ Also see P.-G. de Gennes, Macromolecules 15, 492 (1982).

${ }^{14} \mathrm{~B}$. M. McCoy and T. T. $\mathrm{Wu}$, The Two-Dimensional Ising
Model (Harvard University Press, Cambridge, Massachusetts, 1973).

${ }^{15}$ For example, R. F. M. Houtappel, Physica (Utrecht) 16, 425 (1950).

${ }^{16}$ H. Nakanishi and M. E. Fisher, J. Chem. Phys. 78, 3279 (1983).

${ }^{17}$ In Ref. 5, $B$ was taken equal to $\frac{1}{5}$ instead of $\frac{1}{3}$. The value $\frac{1}{5}$ was derived from the incorrect Eq. (3.10) in Ref. 16, which misses a term $2 S$ in the numerator. Furthermore, $C^{2}$ was taken equal to $1 / 2 d=q^{-1}$ instead of the correct replacement $C^{2}=q^{-2}$.

${ }^{18}$ M. P. Nightingale and J. O. Indekeu, Phys. Rev. Lett. 55, 1700 (1985).

${ }^{19}$ A. A. Migdal, Zh. Eksp. Teor. Fiz. 69, 1457 (1975) [Sov. Phys.-JETP 42, 743 (1976)].

${ }^{20}$ L. P. Kadanoff, Ann. Phys. (N.Y.) 100, 359 (1976).

${ }^{21}$ In principle, the right-hand side of (4.1) should also contain the term $\lim _{k \rightarrow \infty} b^{-d k}\left[-f_{B}\left(K_{B}^{(k)}\right)-d K_{B}^{(k)}\right]$. Analogous terms occur, in principle, in expressions similar to (4.1) for the total slab free energy $F$ and the surface free energy per column $f_{S}$. However, these terms all vanish for all values of the couplings (and fields) provided one properly normalizes the Boltzmann weights initially and after every RG iteration. Otherwise, the RG sum for the free energy is not unique and may converge to an incorrect result. In such cases, one has to invoke an extra term in order to compensate for the error made (Ref. 23).

${ }^{22}$ See, e.g., D. Andelman and J. S. Walker, Phys. Rev. B 27, 241 (1983).

${ }^{23}$ R. Lipowsky and H. Wagner, Z. Phys. B 42, 355 (1981).

${ }^{24} \mathrm{~T}$. W. Burkhardt, in Real-Space Renormalization, edited by T. W. Burkhardt and J. M. J. van Leeuwen (Springer-Verlag, New York, 1982), p. 33.

${ }^{25}$ A. L. Stella, Physica 111A, 513 (1982).

${ }^{26}$ W. V. Wang, Bachelor's thesis, Massachusetts Institute of Technology, 1985.

27J. Stephenson, J. Math. Phys. 5, 1009 (1964).

${ }^{28}$ N. Parga and J. E. Van Himbergen, Ann. Phys. (N.Y.) 134, 286 (1981). 